\title{
Nuclear-matter equation of state with consistent two- and three-body perturbative chiral interactions
}

\author{
L. Coraggio, ${ }^{1}$ J. W. Holt,${ }^{2}$ N. Itaco,,${ }^{1,3}$ R. Machleidt, ${ }^{4}$ L. E. Marcucci,,${ }^{5,6}$ and F. Sammarruca ${ }^{4}$ \\ ${ }^{1}$ Istituto Nazionale di Fisica Nucleare, Complesso Universitario di Monte S. Angelo, Via Cintia, I-80126 Napoli, Italy \\ ${ }^{2}$ Department of Physics, University of Washington, Seattle, Washington 98195, USA \\ ${ }^{3}$ Dipartimento di Fisica, Università di Napoli Federico II, Complesso Universitario di Monte S. Angelo, Via Cintia, I-80126 Napoli, Italy \\ ${ }^{4}$ Department of Physics, University of Idaho, Moscow, Idaho 83844, USA \\ ${ }^{5}$ Dipartimento di Fisica "Enrico Fermi," Università di Pisa, Largo Bruno Pontecorvo 3, I-56127 Pisa, Italy \\ ${ }^{6}$ Istituto Nazionale di Fisica Nucleare, Sezione di Pisa, Largo Bruno Pontecorvo 3, I-56127 Pisa, Italy
}

(Received 5 February 2014; published 24 April 2014)

\begin{abstract}
We compute the energy per particle of infinite symmetric nuclear matter from chiral $\mathrm{N}^{3} \mathrm{LO}$ (next-to-nextto-next-to-leading order) two-body potentials plus $\mathrm{N}^{2} \mathrm{LO}$ three-body forces. The low-energy constants of the chiral three-nucleon force that cannot be constrained by two-body observables are fitted to reproduce the triton binding energy and the ${ }^{3} \mathrm{H}-{ }^{3} \mathrm{He}$ Gamow-Teller transition matrix element. In this way, the saturation properties of nuclear matter are reproduced in a parameter-free approach. The equation of state is computed up to third order in many-body perturbation theory, with special emphasis on the role of the third-order particle-hole diagram. The dependence of these results on the cutoff scale and regulator function is studied. We find that the inclusion of three-nucleon forces consistent with the applied two-nucleon interaction leads to a reduced dependence on the choice of the regulator only for lower values of the cutoff.
\end{abstract}

DOI: 10.1103/PhysRevC.89.044321

PACS number(s): 21.30.Fe, 21.65.Cd, 21.60.Jz

\section{INTRODUCTION}

High-precision nuclear potentials based on chiral perturbation theory (ChPT) [1-3] are nowadays widely employed to link the fundamental theory of strong interactions, quantum chromodynamics (QCD), to nuclear many-body phenomena. An important feature of ChPT is that nuclear two-body forces, many-body forces, and currents [3-5] are generated on an equal footing. Consistency then requires that certain low-energy constants (LECs) appearing in two-nucleon forces (2NF) — and fitted to two-nucleon data-appear also in three-nucleon forces (3NF), four-nucleon forces (4NF), and electroweak currents.

Because ChPT is a low-momentum expansion valid only for momenta $Q<\Lambda_{\chi} \simeq 1 \mathrm{GeV}$, where $\Lambda_{\chi}$ denotes the chiralsymmetry-breaking scale, nucleon-nucleon $(N N)$ potentials derived from ChPT are typically multiplied by a (nonlocal) regulator function,

$$
f\left(p^{\prime}, p\right)=\exp \left[-\left(p^{\prime} / \Lambda\right)^{2 n}-(p / \Lambda)^{2 n}\right],
$$

where $\Lambda \simeq 0.5 \mathrm{GeV}$ is a typical choice for the cutoff scale. In the effective field theory (EFT) framework, the calculated physical observables ideally will be independent of both the regulator function and the associated cutoff scale $\Lambda$. In the case of nuclear interactions this is rarely the case, and varying the regulator is often used as a tool to estimate the uncertainty in the theoretical calculations. In the two-nucleon problem, the dependence of the solutions of the Lippmann-Schwinger equation on the regulator function and its cutoff scale is minimized by a renormalization procedure in which the LECs associated with $N N$ and $\pi N$ vertices are readjusted to two-nucleon phase shifts and deuteron properties. Even though potentials with different regulator functions yield similar phase shifts, they will in general give different predictions when employed in many-body calculations, due to their different off-shell behavior. One is then faced with a larger cutoff dependence in many-body systems [6], which should be reduced by a consistent adjustment of the LECs appearing in nuclear many-body forces.

In a recent paper [7], we have studied the regulator dependence of the cold neutron matter equation of state (EOS) employing chiral two- and three-nucleon potentials within many-body perturbation theory. Previous studies of infinite symmetric nuclear matter and pure neutron matter [8-16] have focused on the importance of nuclear many-body forces and have explored the perturbative and nonperturbative features of chiral nuclear potentials. In Ref. [7] we observed that in neutron matter calculations the use of consistent 3NF plays a crucial role in the restoration of regulator independence. The calculation of the ground-state energy of infinite neutron matter with chiral $3 \mathrm{NF}$ up to $\mathrm{N}^{2} \mathrm{LO}$ depends only on LECs that have been fixed in the two-nucleon system [17]. In the case of symmetric nuclear matter, also the one-pion exchange $3 \mathrm{NF}$ $V_{3 N}^{1 \pi}$ and the contact $3 \mathrm{NF} V_{3 N}^{\text {cont }}$ at $\mathrm{N}^{2} \mathrm{LO}$ contribute. Therefore, the associated LECs $c_{D}$ and $c_{E}$, which are not constrained by two-body observables, must also be refitted for different regulator functions. These $3 \mathrm{NF}$ LECs should be adjusted to $A=3$ observables only, and a possible choice [18-20] is to reproduce the ${ }^{3} \mathrm{H}$ and ${ }^{3} \mathrm{He}$ binding energies together with the triton half-life (specifically the Gamow-Teller matrix element).

In the present work, we continue the investigation started in Ref. [7] and study the dependence of the EOS of symmetric nuclear matter on the choice of regulator function in the chiral nuclear potentials, employing $2 \mathrm{NF}$ and $3 \mathrm{NF}$ with consistent LECs. The ability to obtain realistic nuclear matter predictions with (consistent) two- and three-body interactions constrained by the properties of the two- and the three-nucleon systems and no additional adjustments is a focal point of this paper. 
Historically, this has proven to be a nontrivial task. As in Ref. [7], we employ three different chiral potentials with cutoff scales $\Lambda=414 \mathrm{MeV}$ [21], $450 \mathrm{MeV}$, and $500 \mathrm{MeV}$ [1,3]. The LECs $c_{D}$ and $c_{E}$ of the $\mathrm{N}^{2} \mathrm{LO}$ chiral $3 \mathrm{NF}$ are fitted, for each value of $\Lambda$, to the binding energies of $A=3$ nuclei and the ${ }^{3} \mathrm{H}-{ }^{3} \mathrm{He}$ Gamow-Teller matrix element. Note that the $\Lambda=500 \mathrm{MeV}$ two- and three-nucleon chiral potentials have been used to study $A=3$ and 4 elastic scattering [22], the $A \leqslant 3$ nuclei electromagnetic structure [23], and low-energy reactions of astrophysical interest [24], finding good agreement with the experimental data when available.

We compute the energy per particle of symmetric nuclear matter up to third order in many-body perturbation theory. Previous calculations [13-15,25] beyond second order have focused on the inclusion of particle-particle (pp) and holehole (hh) ladder diagrams, whereas in the present work we compute, in addition to the third-order pp and hh diagrams, also the third-order particle-hole (ph) diagram (without simplifying approximations), which has not been considered previously but is necessary for a consistent third-order calculation. The effects of the $\mathrm{N}^{2} \mathrm{LO} 3 \mathrm{NF}$ are included via a density-dependent twobody potential $\bar{V}_{3 N}$ that is added to the chiral $\mathrm{N}^{3} \mathrm{LO}$ potential $V_{2 N}$ and that is obtained by summing one nucleon over the noninteracting filled Fermi sea of nucleons [17,26,27].

The paper is organized as follows. In Sec. II, we briefly describe the features of the different chiral potentials employed and provide details about the renormalization procedure we have followed to choose the LECs of the $3 \mathrm{NF}$ terms $V_{3 N}^{1 \pi}$ and $V_{3 N}^{\text {cont }}$. In Sec. III, we outline the perturbative calculation of the energy per particle in symmetric nuclear matter that takes into account $3 \mathrm{NF}$ effects. Our results and conclusions are presented in Secs. IV and V, respectively.

\section{SCALE DEPENDENCE OF CHIRAL TWO- AND THREE-NUCLEON POTENTIALS}

During the past two decades, chiral EFT has emerged as a powerful tool for describing hadronic interactions at low-energy scales in a systematic and model-independent way (see Refs. [3,28] for recent reviews). The separation of scales required to construct a useful EFT arises naturally in nuclear interactions from the pseudo-Goldstone boson nature of pions, which is associated with the spontaneous breaking of chiral symmetry and is responsible for the large difference between the light pion mass $\left(m_{\pi} \simeq 135 \mathrm{MeV}\right)$ and the masses of the next lowest states in the meson spectrum, the $\rho(770)$ and $\omega(782)$.

In normal nuclear many-body systems, the nuclear momenta are on the order of the pion mass, and therefore the systematic construction of chiral nuclear potentials is based on an expansion in powers of this soft scale $\left(Q \sim m_{\pi}\right)$ over the hard scale set by the typical hadron masses $\Lambda_{\chi} \sim m_{\rho} \sim 1 \mathrm{GeV}$, also known as the chiral-symmetry-breaking scale. For this EFT to rise above the level of phenomenology, it must have a firm link with QCD. The link is established by having the EFT observe all relevant symmetries of the underlying theory, in particular, the broken chiral symmetry of low-energy QCD [29]. The past 15 years have seen great progress in applying ChPT to nuclear forces. As a result, $N N$ potentials of high precision have been constructed, which are based upon $\mathrm{ChPT}$ carried to $\mathrm{N}^{3} \mathrm{LO}$.

Since ChPT is a low-momentum expansion, valid only for momenta $Q<\Lambda_{\chi}$, the potentials are multiplied with a regulator function, like, e.g., the one of Gaussian shape given in Eq. (1). In this investigation, we consider three $\mathrm{N}^{3} \mathrm{LO} N N$ potentials which differ by the cutoff scale $\Lambda$ and the regulator function. (i) $\Lambda=414 \mathrm{MeV}$ using the regulator function of Eq. (1) with $n=10$, i.e., a smooth, but rather steep cutoff function is applied. This potential is very similar to the one with a sharp cutoff at $414 \mathrm{MeV}$ published in Ref. [21]; however, a smooth version of the steep cutoff is more convenient in calculations of the three-body system. (ii) $\Lambda=450 \mathrm{MeV}$, using the regulator function of Eq. (1) with $n=3$, which has been constructed for our study of Ref. [7] and the present investigation. (iii) $\Lambda=500 \mathrm{MeV}$, using the regulator function of Eq. (1) with $n=2$ for the $2 \pi$ exchange contributions [1]. All three potentials use the same (comprehensive) analytic expressions, which can be found in Ref. [3]. Note that the Gaussian regulator function of Eq. (1) suppresses the potential
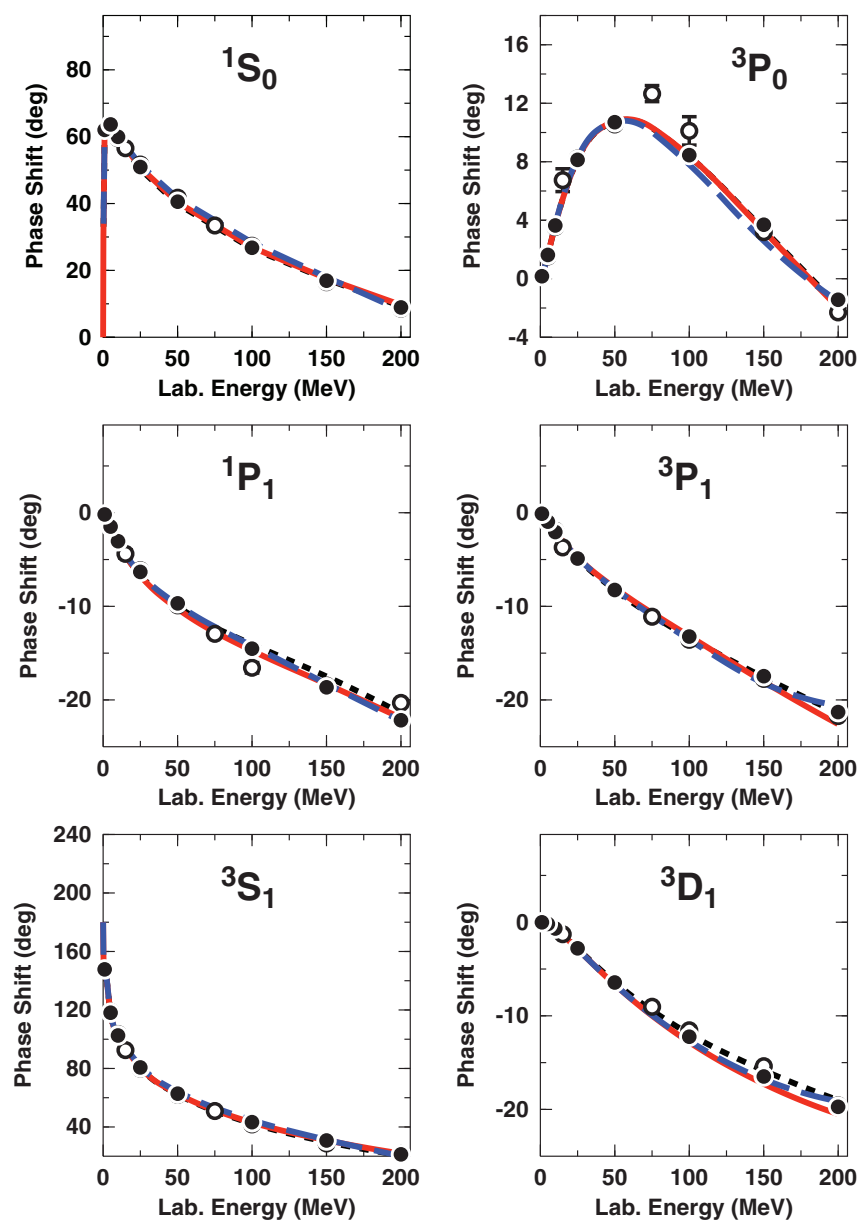

FIG. 1. (Color online) Neutron-proton phase shifts as predicted by chiral $\mathrm{N}^{3} \mathrm{LO}$ potentials with different cutoff scale $\Lambda$. Solid (red) curve, $\Lambda=414 \mathrm{MeV}$; dashed (blue) curve, $\Lambda=450 \mathrm{MeV}$; and dotted (black) curve, $\Lambda=500 \mathrm{MeV}$. Partial waves with total angular momentum $J \leqslant 1$ are displayed. The solid dots and open circles are the results from the Nijmegen multienergy $n p$ phase shift analysis [30] and the VPI/GWU single-energy $n p$ analysis SM99 [31], respectively. 

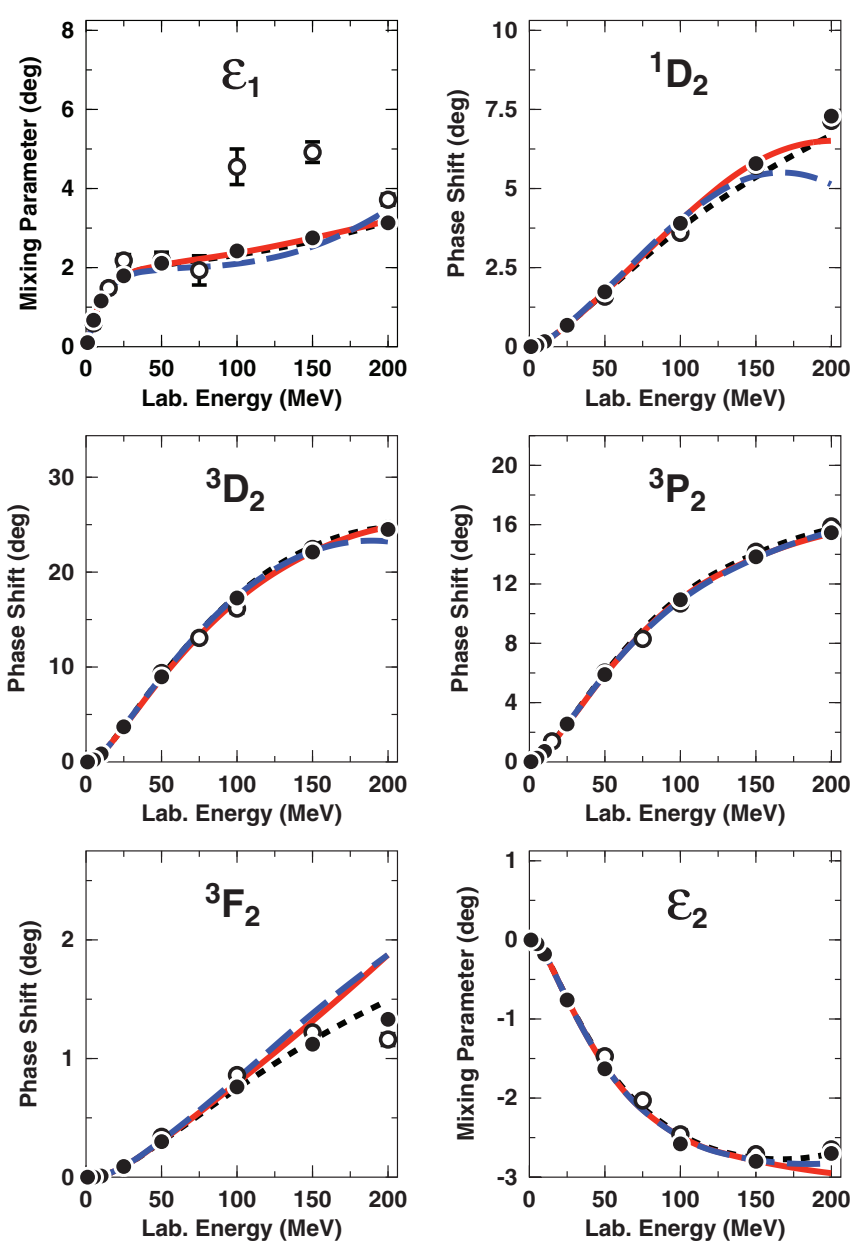

FIG. 2. (Color online) Same as Fig. 1, but for $J=2$ phase shifts and $J \leqslant 2$ mixing parameters.

also for $Q<\Lambda$, particularly for small $n$, which is the reason why we use $n=10$ for the case of the lowest cutoff of $414 \mathrm{MeV}$. Cutoff independence is an important aspect of an EFT. In lower partial waves, the cutoff dependence of the $N N$ phase shifts is counterbalanced by an appropriate adjustment of the contact terms which, at $\mathrm{N}^{3} \mathrm{LO}$, contribute in $S, P$, and $D$ waves. The extent to which cutoff independence can be achieved in lower partial waves is demonstrated in Figs. 1 and 2. In $F$ and higher partial waves (where there are no $N N$ contact terms) the LECs of the dimension-two $\pi N$ Lagrangian can be used to obtain cutoff independence of the phase shift predictions, as shown in Fig. 3.

An important advantage of the EFT approach to nuclear forces is that it creates two- and many-body forces on an equal footing. The first nonvanishing $3 \mathrm{NF}$ occurs at $\mathrm{N}^{2} \mathrm{LO}$. At this order, there are three $3 \mathrm{NF}$ topologies: the two-pion exchange (2PE), one-pion exchange (1PE) plus a $2 N$-contact interaction, and a pure $3 N$-contact interaction. These last two topologies are represented in Fig. 4.

The $2 \mathrm{PE} 3 N$ potential is given by

$$
V_{3 N}^{2 \pi}=\left(\frac{g_{A}}{2 f_{\pi}}\right)^{2} \frac{1}{2} \sum_{i \neq j \neq k} \frac{\left(\vec{\sigma}_{i} \cdot \vec{q}_{i}\right)\left(\vec{\sigma}_{j} \cdot \vec{q}_{j}\right)}{\left(q_{i}^{2}+m_{\pi}^{2}\right)\left(q_{j}^{2}+m_{\pi}^{2}\right)} F_{i j k}^{a b} \tau_{i}^{a} \tau_{j}^{b},
$$
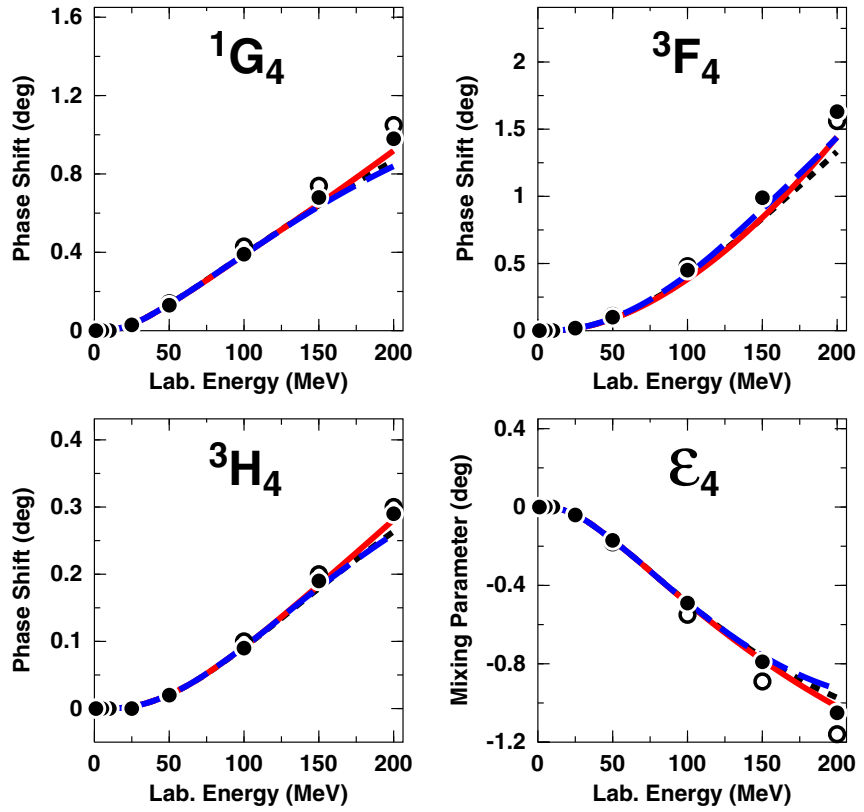

FIG. 3. (Color online) Same as Fig. 1, but for some representative peripheral partial waves.

with $\vec{q}_{i} \equiv \vec{p}_{i}{ }^{\prime}-\vec{p}_{i}$, where $\vec{p}_{i}$ and $\vec{p}_{i}{ }^{\prime}$ are the initial and final momenta of nucleon $i$, respectively, and

$$
\begin{aligned}
F_{i j k}^{a b}= & \delta^{a b}\left[-\frac{4 c_{1} m_{\pi}^{2}}{f_{\pi}^{2}}+\frac{2 c_{3}}{f_{\pi}^{2}} \vec{q}_{i} \cdot \vec{q}_{j}\right] \\
& +\frac{c_{4}}{f_{\pi}^{2}} \sum_{c} \epsilon^{a b c} \tau_{k}^{c} \vec{\sigma}_{k} \cdot\left[\vec{q}_{i} \times \vec{q}_{j}\right] .
\end{aligned}
$$

Note that the $2 \mathrm{PE} 3 \mathrm{NF}$ does not contain any new parameters, because the LECs $c_{1}, c_{3}$, and $c_{4}$ appear already in the $2 \mathrm{PE} 2 \mathrm{NF}$. The 1PE contribution is

$V_{3 N}^{1 \pi}=-\frac{c_{D}}{f_{\pi}^{2} \Lambda_{\chi}} \frac{g_{A}}{8 f_{\pi}^{2}} \sum_{i \neq j \neq k} \frac{\vec{\sigma}_{j} \cdot \vec{q}_{j}}{q_{j}^{2}+m_{\pi}^{2}}\left(\boldsymbol{\tau}_{i} \cdot \boldsymbol{\tau}_{j}\right)\left(\vec{\sigma}_{i} \cdot \vec{q}_{j}\right)$,

and the $3 N$-contact potential reads

$$
V_{3 N}^{\text {cont }}=\frac{c_{E}}{f_{\pi}^{4} \Lambda_{\chi}} \frac{1}{2} \sum_{j \neq k} \boldsymbol{\tau}_{j} \cdot \boldsymbol{\tau}_{k} .
$$

In the above, we use $g_{A}=1.29, f_{\pi}=92.4 \mathrm{MeV}, m_{\pi}=$ $138.04 \mathrm{MeV}$, and $\Lambda_{\chi}=700 \mathrm{MeV}$.

The last two $3 \mathrm{NF}$ terms involve the two new parameters, $c_{D}$ and $c_{E}$, which do not appear in the $2 N$ problem. There are many ways to constrain these two parameters. The triton
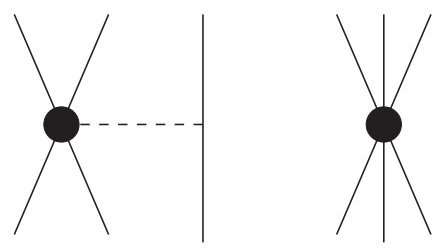

FIG. 4. The $\mathrm{N}^{2} \mathrm{LO}$ three-nucleon force contact interactions: $V_{3 N}^{1 \pi}$ on the left and $V_{3 N}^{\text {cont }}$ on the right [see Eqs. (4) and (5), respectively]. 


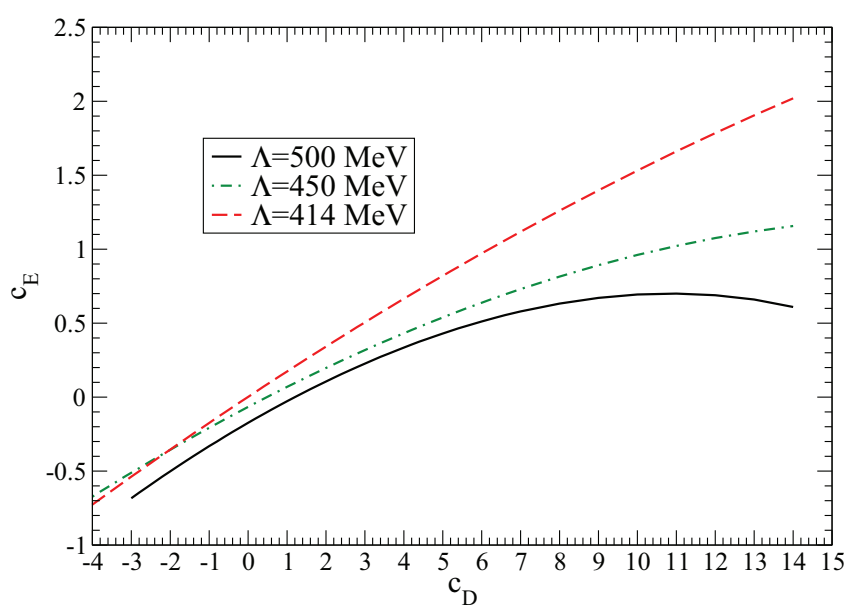

FIG. 5. (Color online) $c_{D}-c_{E}$ trajectories fitted to reproduce the experimental ${ }^{3} \mathrm{H}$ and ${ }^{3} \mathrm{He}$ binding energies. Solid (black) curve for $\Lambda=500 \mathrm{MeV}$, dotted-dashed (green) curve for $\Lambda=450 \mathrm{MeV}$, and dashed (red) curve for $\Lambda=414 \mathrm{MeV}$.

binding energy and the $n d$ doublet scattering length ${ }^{2} a_{n d}$ or the ${ }^{4} \mathrm{He}$ binding energy can be used. Given the known correlation between these observables, one may choose instead an optimal overall fit of the properties of light nuclei [32]. However, recently a new procedure has been used to fix $c_{D}$ and $c_{E}$ [18-20]. Due to the consistency of interactions and currents in chiral EFT $[18,19]$, the LEC $c_{D}$ that appears in $V_{3 N}^{1 \pi}$ is also involved in the two-nucleon contact term in the $N N$ axial current operator derived up to $\mathrm{N}^{2} \mathrm{LO}$. Therefore, $c_{D}$ can be constrained using the accurate experimental value of one observable from weak processes involving two- or few-nucleon systems. Given the lack of accurate experimental values for weak observables in the two-body sector, the choice has been to use the triton $\beta$-decay half-life, in particular its Gamow-Teller (GT) component. This observable has been used already in a variety of studies to constrain the two-body axial current operator [20,24,33-35]. Therefore, we proceed here as in Ref. [20]. (i) We calculate the ${ }^{3} \mathrm{H}$ and ${ }^{3} \mathrm{He}$ wave functions with the hyperspherical harmonics method (see Ref. [36] for a review), using the chiral 2NF plus $3 \mathrm{NF}$ presented above for each cutoff parameter $\Lambda$. The corresponding set of LECs $c_{D}$ and $c_{E}$ are determined by fitting the $A=3$ experimental binding energies. The resulting trajectories are shown in Fig. 5. (ii) For each set of $c_{D}$ and $c_{E}$, the ${ }^{3} \mathrm{H}$ and ${ }^{3} \mathrm{He}$ wave functions are used to calculate the GT matrix element. Comparison with the experimental value leads to a range of values for $c_{D}$ for each cutoff parameter $\Lambda$, as shown in Fig. 6. Then, from Fig. 5, the corresponding range for $c_{E}$ is determined. The values for $c_{D}$ and $c_{E}$ used in the present calculation are listed in Table I for each $\Lambda$. Note that the values of $c_{D}$ and $c_{E}$ for $\Lambda=500 \mathrm{MeV}$ are slightly different from those used in previous studies [20,22,24], but the GT matrix element is still reproduced within less than $1 \%$.

At this point, a final remark is in order: the present fitting procedure employs $\mathrm{N}^{3} \mathrm{LO} N N$ interaction together with a $3 \mathrm{NF}$ derived at $\mathrm{N}^{2} \mathrm{LO}$, i.e., one chiral order lower. Furthermore, also the weak current operator is derived at

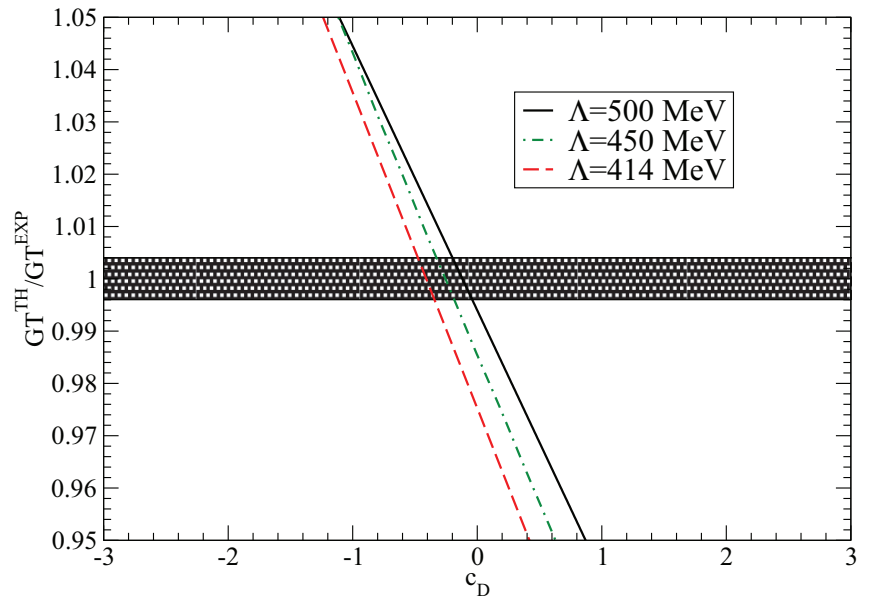

FIG. 6. (Color online) The ratio between the calculated GT value $\left(\mathrm{GT}^{\mathrm{TH}}\right)$ and the experimental one $\left(\mathrm{GT}^{\mathrm{EXP}}\right)$ as a function of the LEC $c_{D}$. Solid (black) curve for $\Lambda=500 \mathrm{MeV}$, dotted-dashed (green) curve for $\Lambda=450 \mathrm{MeV}$, and dashed (red) curve for $\Lambda=414 \mathrm{MeV}$. The shaded stripe represents the experimental uncertainty.

$\mathrm{N}^{2}$ LO. To avoid this mismatch, it would be necessary to use $\mathrm{N}^{3} \mathrm{LO}$ two- and three-nucleon interactions and currents. However, a derivation at $\mathrm{N}^{3} \mathrm{LO}$ of the electroweak current would require the calculation of loop corrections, a task successfully performed for the electromagnetic operators [23], but not yet for the axial operators. Furthermore, the $3 \mathrm{NF}$ at $\mathrm{N}^{3} \mathrm{LO}$ has been derived only recently $[37,38]$ and, due to its complexity, only preliminary studies have been performed so far $[16,39,40]$.

\section{NUCLEAR MATTER CALCULATIONS}

We calculate the ground-state energy (g.s.e.) per particle of infinite symmetric nuclear matter within the framework of many-body perturbation theory. More precisely, the g.s.e. is expressed as a sum of Goldstone diagrams up to third order.

As mentioned in Sec. I, the effects of the 3NF are taken into account via a density-dependent two-body potential, $\bar{V}_{3 N}$,

TABLE I. For the various chiral $\mathrm{N}^{3} \mathrm{LO} N N$ potentials used in the present investigation, we list the cutoff $\Lambda$, the type of regulator, the exponent $n$ used in the regulator function [see Eq. (1)], the LECs of the dimension-two $\pi N$ Lagrangian, $c_{i}$ (in units of $\mathrm{GeV}^{-1}$ ), and the LECs $c_{D}$ and $c_{E}$ entering the three-nucleon potential.

\begin{tabular}{lccc}
\hline \hline & \multicolumn{3}{c}{ Cutoff parameter $\Lambda(\mathrm{MeV})$} \\
\cline { 2 - 4 } & 414 & 450 & 500 \\
\hline Regulator type & Gaussian & Gaussian & Gaussian \\
$n$ & 10 & 3 & 2 \\
$c_{1}$ & -0.81 & -0.81 & -0.81 \\
$c_{2}$ & 3.28 & 3.28 & 2.80 \\
$c_{3}$ & -3.00 & -3.40 & -3.20 \\
$c_{4}$ & 3.40 & 3.40 & 5.40 \\
$c_{D}$ & -0.40 & -0.24 & 0.0 \\
$c_{E}$ & -0.07 & -0.11 & -0.18 \\
\hline \hline
\end{tabular}




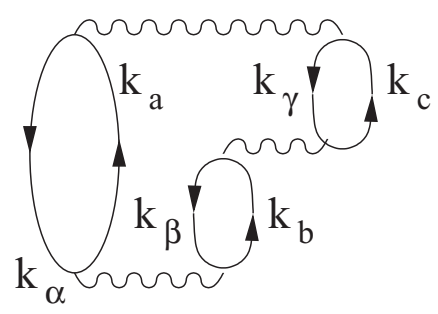

FIG. 7. Third-order ring diagram of the Goldstone expansion that we have included in our calculations with $V_{2 N}$ and $\bar{V}_{3 N}$ vertices. Latin-letter subscripts denote particle states; Greek-letter subscripts correspond to hole states.

that is added to the chiral $\mathrm{N}^{3} \mathrm{LO}$ potential $V_{2 N}$. This potential is obtained by summing one nucleon over the occupied states in the (non-interacting) filled Fermi sea, thus leading to a density-dependent two-nucleon interaction $\bar{V}_{3 N}\left(k_{F}\right)$. At this time, analytic expressions for $\bar{V}_{3 N}[26,27]$ have been derived only for the $\mathrm{N}^{2} \mathrm{LO} 3 \mathrm{NF}$, which is the one we take into account in this work. We recall that to take care of the correct combinatorial factors of the normal-ordering at the two-body level of the $3 \mathrm{NF}$, the matrix elements of $\bar{V}_{3 N}\left(k_{F}\right)$ have to be multiplied by a factor $1 / 3$ in the first-order Hartree-Fock (HF) diagram and by a factor $1 / 2$ in the calculation of the HF single-particle energies [7,17].

We point out that in the present calculations we have summed the perturbation expansion up to third-order in $V_{2 N}+\bar{V}_{3 N}$, in particular, including the third-order particlehole (ph) diagram (see Fig. 7), which is also known as the third-order ring diagram [41]. This diagram has been taken into account neither in our previous paper [7] nor in other recent nuclear matter calculations which have employed chiral potentials within a perturbative approach $[8,9,11,15]$. The analytic expressions of first-, second-, and third-order particle-particle (pp) and hole-hole (hh) contributions, together with the one of single-particle HF potential, have been already reported in Ref. [7]. The implicit expression of the third-order ph diagram can be found in Ref. [42], where also the explicit expressions for a potential without tensor and spin-orbit forces are reported.

The contributions of each diagram to the perturbation expansion obtained with the three chiral potentials for $k_{F}=$ $1.3 \mathrm{fm}^{-1}$ without and with $3 \mathrm{NF}$ effects are given in Tables II and III, respectively. It is clear that the magnitude of the

TABLE II. Contributions of each diagram to the perturbation expansion (in $\mathrm{MeV}$ ) obtained with the three chiral potentials for $k_{F}=1.3 \mathrm{fm}^{-1}$ taking into account only $2 \mathrm{NFs}$.

\begin{tabular}{lrrr}
\hline \hline & \multicolumn{3}{c}{ Cutoff parameter $\Lambda(\mathrm{MeV})$} \\
\cline { 2 - 4 } & \multicolumn{1}{c}{414} & \multicolumn{1}{c}{450} & \multicolumn{1}{c}{500} \\
\hline HF contribution & -35.507 & -32.786 & -25.066 \\
Second-order pp diagram & -5.736 & -8.551 & -14.060 \\
Third-order pp diagram & 0.017 & -0.022 & 0.653 \\
Third-order hh diagram & -0.022 & -0.021 & -0.027 \\
Third-order ph diagram & 1.040 & 1.200 & -0.279 \\
\hline \hline
\end{tabular}

TABLE III. Same as in Table II, but including also 3NF effects.

\begin{tabular}{lrrr}
\hline \hline & \multicolumn{3}{c}{ Cutoff parameter $\Lambda(\mathrm{MeV})$} \\
\cline { 2 - 4 } & \multicolumn{1}{c}{414} & \multicolumn{1}{c}{450} & \multicolumn{1}{c}{500} \\
\hline HF contribution & -28.792 & -25.688 & -19.503 \\
Second-order pp diagram & -7.388 & -11.273 & -13.511 \\
Third-order pp diagram & 0.563 & 0.745 & 1.642 \\
Third-order hh diagram & -0.010 & -0.008 & -0.008 \\
Third-order ph diagram & 0.581 & 0.152 & -1.516 \\
\hline \hline
\end{tabular}

third-order ph diagram is large, bringing a relevant contribution to the third-order energy.

This is in line with the results shown in Ref. [16], where the neutron and nuclear EOS have been calculated within the coupled-cluster approach employing the chiral $\mathrm{NNLO}_{\text {opt }}$ potential [43]. As a matter of fact, in Ref. [16] the inclusion of perturbative triples corrections in the coupledcluster equations leads to corrections for the binding energy of about $1 \mathrm{MeV}$ per nucleon, when including the $3 \mathrm{NF}$ in the normal-ordered two-body approximation. Furthermore, it is insightful to note that in Ref. [16] a significant contribution was found when going beyond the 3NF normal-ordered two-body approximation. With that in mind, we estimate the uncertainty of our perturbative result to be approximately $2 \mathrm{MeV}$.

To study the convergence properties of the perturbative expansion, it is useful to consider the [2|1] Padé approximant [44]

$$
E_{[2 \mid 1]}=\mathcal{E}_{0}+\mathcal{E}_{1}+\frac{\mathcal{E}_{2}}{1-\mathcal{E}_{3} / \mathcal{E}_{2}},
$$

$\mathcal{E}_{i}$ being the $i$ th order energy contribution in the perturbative expansion of the g.s.e.. The Padé approximant is an estimate of the value to which the perturbative series may converge. Thus, in the following section we perform a comparison between the third-order results and those obtained by means of the [2|1] Padé approximant to obtain an indication of the size of the higher-order perturbative terms.

\section{RESULTS}

In this section, we report the results of the calculation of the EOS of infinite symmetric nuclear matter in the framework of many-body perturbation theory. Because we include all contributions up to third order in the interaction, we are in a good position to study the convergence properties of the perturbative expansion.

We find that among the three chiral potentials under consideration, the least satisfactory perturbative behavior belongs to the chiral $\mathrm{N}^{3} \mathrm{LO} N N$ potential with $\Lambda=500 \mathrm{MeV}$, whether the corresponding $\mathrm{N}^{2} \mathrm{LO} 3 \mathrm{NF}$ is included or not. This feature was already observed in our study of pure neutron matter [7] and is apparent in Figs. 8 and 9. In Fig. 8 we show the EOS as a function of the Fermi momentum $k_{F}$, calculated at various orders in the perturbative expansion applying the chiral $\mathrm{N}^{3} \mathrm{LO} N N$ potential with $\Lambda=500 \mathrm{MeV}$. By inspection of the figure, it can be seen that the energy per nucleon calculated at second order, $E_{2}$, does not differ much 


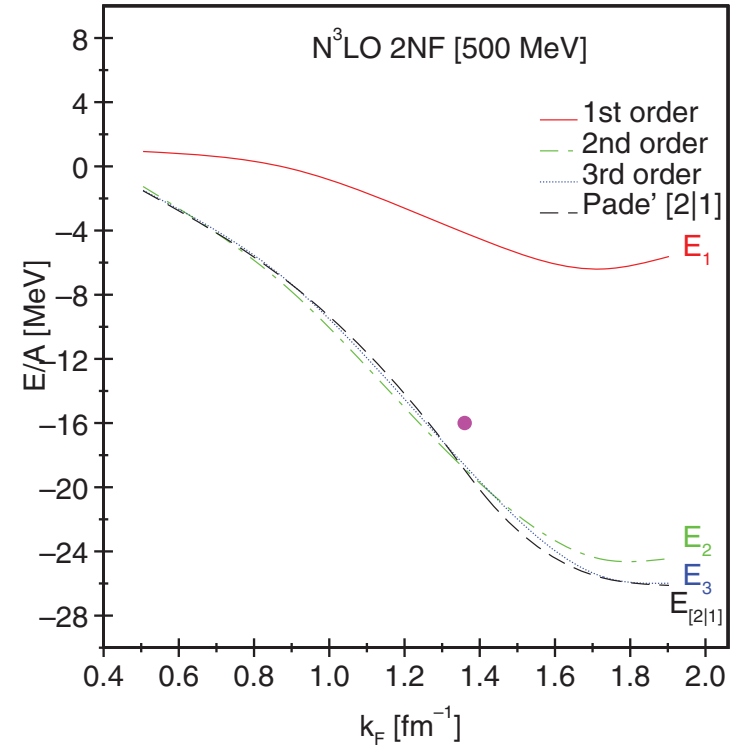

FIG. 8. (Color online) Nuclear matter energy per particle obtained from the $\mathrm{N}^{3} \mathrm{LO} 2 \mathrm{NF}$ with cutoff $\Lambda=500 \mathrm{MeV}$. The first, second, and third order in the perturbative expansion and the Padé approximant [2|1] are shown as a function of the Fermi momentum $k_{F}$.

from the one computed at third order, $E_{3}$, for the whole range of Fermi momenta considered. The perturbative character is also indicated by the fact that the curve corresponding to $E_{3}$ is almost indistinguishable from the [2|1] Padé approximant one.

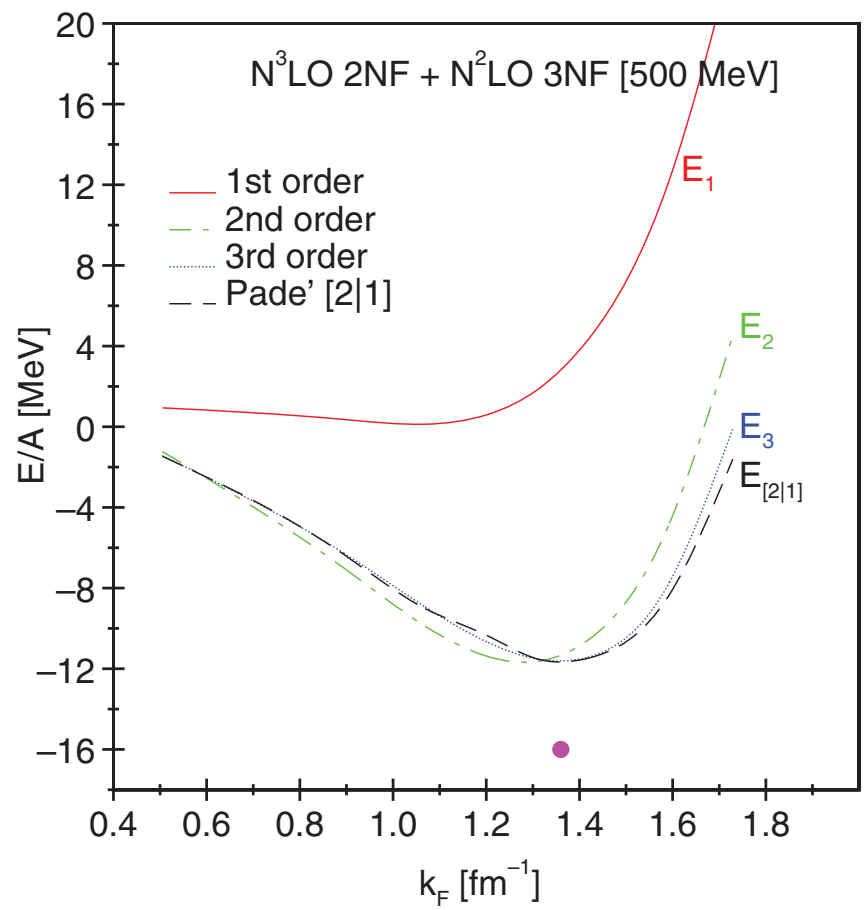

FIG. 9. (Color online) Same as in Fig. 8, but including the contribution of the $\mathrm{N}^{2} \mathrm{LO} 3 \mathrm{NF}$.

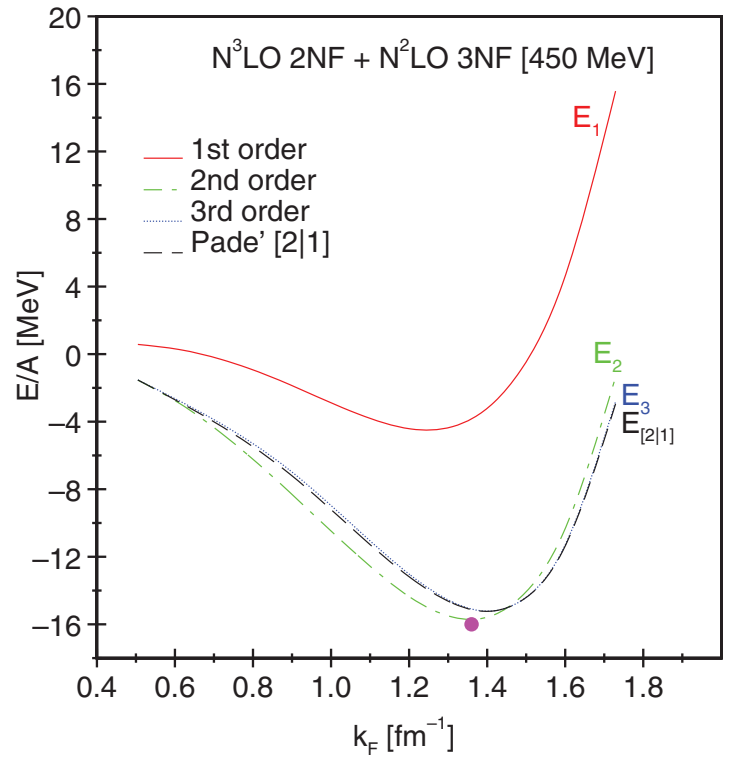

FIG. 10. (Color online) Same as Fig. 9, but for $\Lambda=450 \mathrm{MeV}$.

Different considerations about the perturbative expansion have to be drawn when including the effects of 3NF. As a matter of fact, from inspection of Fig. 9, it can be seen that now the curve corresponding to $E_{3}$ deviates from the one given by the [2|1] Padé approximant for $k_{F}$ larger than $1.6 \mathrm{fm}^{-1}$, indicating a worsening of the perturbative behavior. On the other hand, using the other chiral potentials with lower cutoffs, the perturbative behavior is satisfactory at least up to $k_{F}=1.8$ $\mathrm{fm}^{-1}$, as shown in Fig. 10 for $\Lambda=450 \mathrm{MeV}$.

In Fig. 11 we display our predicted EOS obtained with chiral potentials that apply different regulator functions. We have added to each $2 \mathrm{NF}$ a $3 \mathrm{NF}$ whose LECs $c_{i}$, cutoff

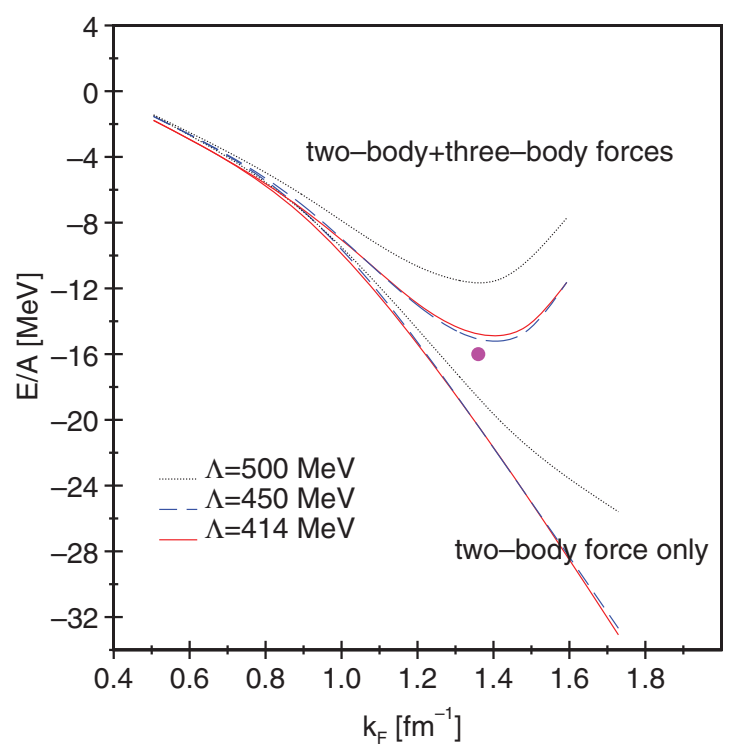

FIG. 11. (Color online) Results obtained for the g.s.e. per particle of infinite nuclear matter at third-order in perturbation theory for three sets of chiral interactions which differ by the cutoff $\Lambda$. 


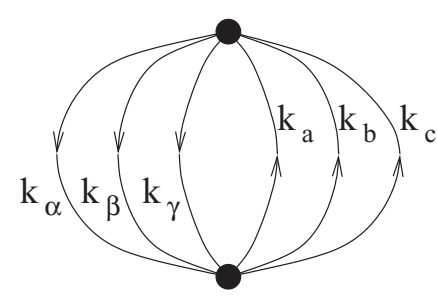

FIG. 12. Second-order Hugenholtz 3p-3h diagram of the Goldstone expansion with two $3 \mathrm{NF}$ vertices. Latin-letter subscripts denote particle states; Greek-letter subscripts correspond to hole states.

parameters, and regulator function are exactly the same as in the corresponding $\mathrm{N}^{3} \mathrm{LO} N N$ potential (see Table I), while the $c_{D}$ and $c_{E}$ LECs have been chosen so as to reproduce the observed $A=3$ binding energies and the triton GT matrix element (see Sec. II). Our results have been obtained at third-order in the perturbative expansion, with and without taking into account $3 \mathrm{NF}$ effects.

The EOS calculated with 2NFs only and cutoffs $\Lambda=414$ and $450 \mathrm{MeV}$ are very close to each other, while the one corresponding to $\Lambda=500 \mathrm{MeV}$ is very different from the others. None of them show saturation, at least up to $k_{F}=$ $1.9 \mathrm{fm}^{-1}$. The differences between the predictions obtained with the two lower cutoffs on the one hand and with the larger cutoff on the other are not removed when including three-body effects. As a matter of fact, while the $\Lambda=414$ and $450 \mathrm{MeV}$ EOS are nearly identical and show realistic saturation properties, the $\Lambda=500 \mathrm{MeV}$ EOS is considerably more repulsive. This is quite different from what we observed in pure neutron matter, where the inclusion of $3 \mathrm{NF}$ effects resulted in a (net) strong regulator-dependence reduction, with the predictions from the three potentials approaching one another.

Despite the fact that the regulator dependence is not removed, the ability to obtain good saturation properties in a microscopic calculation, where the parameters are determined via the few-nucleon systems, should not be underestimated.

Another observation from this study is that the $3 \mathrm{NF}$ contribution to the energy per nucleon in symmetric nuclear matter is larger than that in pure neutron matter [7] (about a factor of 2 at $k_{F}=1.35 \mathrm{fm}^{-1}$ for the $\Lambda=500 \mathrm{MeV}$ case). This may suggest that the weight of $3 \mathrm{p}-3 \mathrm{~h}$ perturbative contributions induced by $3 \mathrm{NF}$ only (which are shown in Fig. 12 and are not included here but come into play at second order and beyond) could be non-negligible.

\section{CONCLUDING REMARKS AND OUTLOOK}

In this paper we have studied the regulator dependence of many-body predictions of the EOS of symmetric nuclear mat- ter, when employing chiral two- and three-nucleon potentials. This has been done within the framework of the perturbative Goldstone expansion and using three different cutoffs and regulator functions for the derivation of the chiral potentials. We have adopted a consistent choice of the LECs and of the regulator functions for the two- and three-body components of the potential. In particular, the LECs $c_{D}$ and $c_{E}$ present in the $3 \mathrm{NF}$ have been fixed so as to reproduce the experimental $A=3$ binding energies and GT matrix element in tritium $\beta$ decay.

Our calculations of the symmetric nuclear matter EOS show that, when employing chiral potentials with cutoffs $\Lambda=414$ and $450 \mathrm{MeV}$, the regulator independence provided by the renormalization procedure for the $A \leqslant 3$ systems is preserved. We note again that these two potentials are found to exhibit good perturbative behavior. Moreover, the introduction of $3 \mathrm{NF}$ effects proves to be crucial for saturation and the predicted saturation properties are consistent with the empirical ones, within the uncertainty estimated in Sec. III. As mentioned above, this is a significant point, as it gives confidence in an $a b$ initio approach with $2 \mathrm{NF}$ and $3 \mathrm{NF}$ consistent with each other and with the properties of few-nucleon systems.

In a previous work [7], where the same topic has been studied for the pure neutron-matter EOS, we have found that the inclusion of $3 \mathrm{NF}$ effects is crucial to restore the above regulator independence also when employing the larger cutoff $\Lambda=500 \mathrm{MeV}$ potential. This is not the case, at least within a perturbative approach, in symmetric nuclear matter, where the $\Lambda=500 \mathrm{MeV}$ EOS is less attractive than the other two by 3 $\mathrm{MeV}$ per nucleon around $k_{F}=1.35 \mathrm{fm}^{-1}$.

From the observation made in the previous section about the relative sizes of $3 \mathrm{NF}$ contributions in nuclear vs neutron matter, we conclude that a calculation of the second-order $3 \mathrm{p}-3 \mathrm{~h}$ diagram may shed light on whether the regulator dependence we have found is an issue with the perturbative expansion or with higher-order terms in ChPT, i.e., $3 \mathrm{NF}$ and $4 \mathrm{NF}$ at $\mathrm{N}^{3} \mathrm{LO}$ $[37,38,45,46]$.

The inclusion of the diagram in Fig. 12 will be a topic of future studies and may provide a better understanding of the application of chiral interactions in microscopic nuclear structure calculations.

\section{ACKNOWLEDGMENTS}

This work was supported in part by the U.S. Department of Energy under Grants No. DE-FG02-03ER41270 and No. DE-FG02-97ER-41014. We thank Norbert Kaiser for helpful discussions concerning the calculation of the third-order particle-hole diagram.
[1] D. R. Entem and R. Machleidt, Phys. Rev. C 68, 041001(R) (2003).

[2] E. Epelbaum, W. Glöckle, and U.-G. Meissner, Nucl. Phys. A 747, 362 (2005).

[3] R. Machleidt and D. R. Entem, Phys. Rep. 503, 1 (2011).
[4] S. Weinberg, Phys. Lett. B 295, 114 (1992).

[5] U. van Kolck, Phys. Rev. C 49, 2932 (1994).

[6] L. Coraggio, A. Covello, A. Gargano, N. Itaco, and T. T. S. Kuo, Ann. Phys. 327, 2125 (2012). 
[7] L. Coraggio, J. W. Holt, N. Itaco, R. Machleidt, and F. Sammarruca, Phys. Rev. C 87, 014322 (2013).

[8] F. Sammarruca, B. Chen, L. Coraggio, N. Itaco, and R. Machleidt, Phys. Rev. C 86, 054317 (2012).

[9] I. Tews, T. Krüger, K. Hebeler, and A. Schwenk, Phys. Rev. Lett. 110, 032504 (2013).

[10] J. W. Holt, N. Kaiser, and W. Weise, Prog. Part. Nucl. Phys. 73, 35 (2013).

[11] T. Krüger, I. Tews, K. Hebeler, and A. Schwenk, Phys. Rev. C 88, 025802 (2013).

[12] A. Gezerlis, I. Tews, E. Epelbaum, S. Gandolfi, K. Hebeler, A. Nogga, and A. Schwenk, Phys. Rev. Lett. 111, 032501 (2013).

[13] A. Carbone, A. Polls, and A. Rios, Phys. Rev. C 88, 044302 (2013).

[14] G. Baardsen, A. Ekström, G. Hagen, and M. Hjorth-Jensen, Phys. Rev. C 88, 054312 (2013).

[15] M. Kohno, Phys. Rev. C 88, 064005 (2013).

[16] G. Hagen, T. Papenbrock, A. Ekström, K. A. Wendt, G. Baardsen, S. Gandolfi, M. Hjorth-Jensen, and C. J. Horowitz, Phys. Rev. C 89, 014319 (2014).

[17] K. Hebeler and A. Schwenk, Phys. Rev. C 82, 014314 (2010).

[18] A. Gårdestig and D. R. Phillips, Phys. Rev. Lett. 96, 232301 (2006).

[19] D. Gazit, Phys. Lett. B 666, 472 (2008).

[20] L. E. Marcucci, A. Kievsky, S. Rosati, R. Schiavilla, and M. Viviani, Phys. Rev. Lett. 108, 052502 (2012).

[21] L. Coraggio, A. Covello, A. Gargano, N. Itaco, D. R. Entem, T. T. S. Kuo, and R. Machleidt, Phys. Rev. C 75, 024311 (2007).

[22] M. Viviani, L. Girlanda, A. Kievsky, and L. E. Marcucci, Phys. Rev. Lett. 111, 172302 (2013).

[23] M. Piarulli, L. Girlanda, L. E. Marcucci, S. Pastore, R. Schiavilla, and M. Viviani, Phys. Rev. C 87, 014006 (2013).

[24] L. E. Marcucci, R. Schiavilla, and M. Viviani, Phys. Rev. Lett. 110, 192503 (2013).

[25] K. Hebeler, S. K. Bogner, R. J. Furnstahl, A. Nogga, and A. Schwenk, Phys. Rev. C 83, 031301(R) (2011).

[26] J. W. Holt, N. Kaiser, and W. Weise, Phys. Rev. C 79, 054331 (2009).

[27] J. W. Holt, N. Kaiser, and W. Weise, Phys. Rev. C 81, 024002 (2010).
[28] E. Epelbaum, H.-W. Hammer, and U.-G. Meißner, Rev. Mod. Phys. 81, 1773 (2009).

[29] S. Weinberg, Phys. A 96, 327 (1979).

[30] V. G. J. Stoks, R. A. M. Klomp, M. C. M. Rentmeester, and J. J. de Swart, Phys. Rev. C 48, 792 (1993).

[31] R. A. Arndt, I. I. Strakovsky, and R. L. Workman, Said Scattering Analysis Interactive Dial-in Computer Facility, George Washington University (formerly Virginia Polytechnic Institute), Solution SM99 (Summer 1999).

[32] P. Navrátil, V. G. Gueorguiev, J. P. Vary, W. E. Ormand, and A. Nogga, Phys. Rev. Lett. 99, 042501 (2007).

[33] L. E. Marcucci, R. Schiavilla, M. Viviani, A. Kievsky, S. Rosati, and J. F. Beacom, Phys. Rev. C 63, 015801 (2000).

[34] T.-S. Park, L. E. Marcucci, R. Schiavilla, M. Viviani, A. Kievsky, S. Rosati, K. Kubodera, D.-P. Min, and M. Rho, Phys. Rev. C 67, 055206 (2003).

[35] L. E. Marcucci, M. Piarulli, M. Viviani, L. Girlanda, A. Kievsky, S. Rosati, and R. Schiavilla, Phys. Rev. C 83, 014002 (2011).

[36] A. Kievsky, S. Rosati, M. Viviani, L. E. Marcucci, and L. Girlanda, J. Phys. G: Nucl. Part. Phys. 35, 063101 (2008).

[37] V. Bernard, E. Epelbaum, H. Krebs, and U.-G. Meißner, Phys. Rev. C 77, 064004 (2008).

[38] V. Bernard, E. Epelbaum, H. Krebs, and U.-G. Meißner, Phys. Rev. C 84, 054001 (2011).

[39] R. Skibinski, J. Golak, K. Topolnicki, H. Witala, E. Epelbaum et al., Few-Body Syst. 54, 1315 (2013).

[40] H. Witala, J. Golak, R. Skibinski, K. Topolnicki, H. Kamada et al., Few-Body Syst. 54, 897 (2013).

[41] H. A. Bethe, Phys. Rev. 138, B804 (1965).

[42] J. J. MacKenzie, Phys. Rev. 179, 1002 (1969).

[43] A. Ekström, G. Baardsen, C. Forssén, G. Hagen, M. HjorthJensen, G. R. Jansen, R. Machleidt, W. Nazarewicz, T. Papenbrock, J. Sarich et al., Phys. Rev. Lett. 110, 192502 (2013).

[44] G. A. Baker and J. L. Gammel, The Padé Approximant in Theoretical Physics, Mathematics in Science and Engineering Vol. 71 (Academic Press, New York, 1970).

[45] E. Epelbaum, Phys. Lett. B 639, 456 (2006).

[46] S. Ishikawa and M. R. Robilotta, Phys. Rev. C 76, 014006 (2007) 